a $1 \mathrm{~h}$ infusion on day 4 . This cycle was repeated a median of four times per patient.

A total of 42 patients were included in the study. The maximum tolerated dose of the drug combination (based on cycle 1) was $1.50 \mathrm{mg} / \mathrm{m}^{2}$ of bortezomib and $30 \mathrm{mg} / \mathrm{m}^{2}$ of pegylated liposomal doxorubicin. Due to frequent dose delays and dose reductions in later cycles, however, the bortezomib dose recommended for subsequent investigations was $1.30 \mathrm{mg} / \mathrm{m}^{2}$.

Of 22 evaluable patients with multiple myeloma, complete remission (or near-complete remission) was seen in eight cases and a further eight patients achieved a partial response. One patient with relapsed/refractory T-cell non-Hodgkin lymphoma achieved a complete response, and antitumor activity was also seen in two patients with acute myeloid leukemia and two patients with B-cell non-Hodgkin lymphoma.

This drug combination is currently undergoing further clinical testing in patients with multiple myeloma, both in the first-line setting, and as a therapy for relapsed/refractory disease.

Original article Orlowski RZ et al. (2005) Phase I trial of the proteasome inhibitor bortezomib and pegylated liposomal doxorubicin in patients with advanced hematologic malignancies. Blood 105: 3058-3065

\section{Biochemical failure following prostate cancer treatment}

Buyyounouski and colleagues from the Fox Chase Cancer Center in Philadelphia have examined the manner in which PSA levels rise and fall following prostate cancer treatment. Their findings indicate that use of the American Society of Therapeutic Radiation Oncology (ASTRO) consensus definition overestimates biochemical failure in a high proportion of patients receiving radiation therapy plus androgen deprivation.

The team studied 586 patients who received radiation therapy alone and 102 patients who underwent radiation therapy and either short-term or long-term androgen deprivation. Post-treatment PSA levels were measured approximately every 6 months during the median follow-up of 75 months. Patients were then further subdivided according to their biochemical failure status, using the ASTRO consensus definition.
Of those patients assigned to the biochemical failure group on the basis of the ASTRO definition, subsequent decline and stablilization of PSA level was observed in $9(22 \%)$ of those receiving short-term androgen-deprivation plus radiotherapy, 2 (29\%) of those receiving longterm androgen-deprivation plus radiotherapy and $10(5 \%)$ of those in the radiotherapyonly group.

In summary, the study showed that biochemical failure was overestimated in $20-30 \%$ of patients treated with radiation therapy and androgen deprivation, and in 5\% of those treated with radiation therapy alone. Commenting that the variation shown between these two groups reflects differences in the temporal kinetics of PSA following treatment, the authors call for the ASTRO definition of biochemical failure to be modified.

Original article Buyyounouski MK et al. (2005) Biochemical failure and the temporal kinetics of prostate-specific antigen after radiation therapy with androgen deprivation. Int $J$ Radiation Oncology Biol Phys 61: 1291-1298

\section{Gemcitabine plus capecitabine in biliary cancer}

Biliary cancer has a poor prognosis. Surgical resection is a potential cure; however, only $25 \%$ of patients are resectable at presentation and relapse rates are high. The median survival time for unresectable biliary cancer is $<1$ year. There is currently no standard chemotherapy that can clearly prolong survival, but newer chemotherapies such as gemcitabine, newer fluorouracil regimens, capecitabine and platinum analogs seem to be active. Combinations of newer drugs may be more active still.

In this single-institute trial in 45 patients with locally advanced or metastatic biliary cancer, patients received a three-week cycle of oral capecitabine $650 \mathrm{mg} / \mathrm{m}^{2}$ twice-daily for 14 days with 30-minute intravenous gemcitabine $1000 \mathrm{mg} / \mathrm{m}^{2}$ on days 1 and 8 of the cycle. 23 patients had cholangiocarcinoma and 22 had gallbladder cancer.

The overall response rate was $31 \%$ and disease stabilization was $42 \%$, giving an overall disease-control rate of $73 \%$. Median progression-free survival for disease-controlled patients was 10 months, and median overall survival was 14 months. Overall response rates for 